\title{
Recovery of Chronic Noise Exposure Induced Spatial Learning and Memory Deficits in Young Male Sprague-Dawley Rats
}

\author{
Chen Chengzhi ${ }^{1 \#}$, Tang Yan ${ }^{2 \#}$, Jiang Xuejun ${ }^{3}$, Li Xiang ${ }^{2}$, Qi Youbin ${ }^{1}$ and Tu Baijie ${ }^{1}$ \\ ${ }^{1}$ Department of Occupational and Environmental Medicine, School of Public Health, Chongqing Medical University, \\ ${ }^{2}$ Department of Occupational and Environmental Medicine, School of Public Health, Luzhou Medical College and \\ ${ }^{3}$ Department of Environmental Health, West China College of Public Health, Sichuan University, China
}

\begin{abstract}
Recovery of Chronic Noise Exposure Induced Spatial Learning and Memory Deficits in Young Male Sprague-Dawley Rats: Chen CHENGzHI, et al. Department of Occupational and Environmental Medicine, School of Public Health, Chongqing Medical University, China-Objective: Noise has always been an important environmental public health issue for mankind. Although reports exist on hippocampaldependent spatial learning and memory (LM) impairment due to noise, there are only a few studies that have examined the effects of recovery from noise stress on LM impairments. Therefore, the present study investigated the effects of recovery from chronic noise exposure on LM deficits. Materials and Methods: In this study, the detrimental effects of noise stress on young male rats in the Morris water maze (MWM) were investigated at $1,10,20,30$, and 40 days after the discontinuation of chronic noise exposure of $80 \mathrm{~dB}$ or $100 \mathrm{~dB}$ for $4 \mathrm{~h}$ per day, for 30 days. The levels of monoamine neurotransmitters in the hippocampus were also evaluated by HPLC-EC at the end of each behavioral test. Results: Statistical analysis revealed that rats in the noise-treated groups failed to reach the same level of performance as the controls in the MWM. Further, the levels of dopamine (DA), norepinephrine (NE) and 5-hydroxytryptamine (5-HT) were significantly decreased in the hippocampus after noise exposure. However, the reduction in monoamine levels and impaired water maze performance recovered over time, so that by 30th day after cessation of noise exposure the $80 \mathrm{~dB}$ group showed no performance difference from the controls, and by 40th day, the $100 \mathrm{~dB}$ group also showed no performance difference from the control.
\end{abstract}

Received Sep 10, 2010; Accepted Feb 1, 2011

Published online in J-STAGE Mar 16, 2011

Correspondence to: T. Yan, Department of Occupational and Environmental Medicine, School of Public Health, Luzhou Medical College, Luzhou, 646000, China

(e-mail:Tangyan200310@yahoo.com.cn)

\# These authors contributed equally to this work.
Conclusion: Our findings suggest that noise impaired LM in young male rats and reduced monoamine neurotransmitters in the hippocampus. However, the noise-induced water maze deficits recovered over time, and the concurrent restoration of hippocampal monoamine neurotransmitter levels suggest that they are involved in LM impairments. (J Occup Health 2011; 53: 157-163)

Key words: Hippocampus, Monoamine neurotransmitter, Morris water maze, Noise, Spatial learning and memory

With rapid industrialization throughout the world, noise pollution has been on an ever-increasing trend, and it is recognized as a serious health problem in modern society. Deleterious effects of noise on various cognitive abilities among adults, such as short-term or long-term memory and mental health, have been demonstrated in previous studies $^{1-4)}$. Exposure to environmental noise was also found to impair a number of cognitive parameters among children $^{5-7)}$. Recently, Clark et al. established the adverse effect of chronic aircraft and road traffic noise exposure on reading comprehension among primary school children ${ }^{8)}$. While some studies in this field have reported difficulties in school learning among children living in noisy areas ${ }^{9,10)}$, relationships between LM problems and chronic noise exposure have not been clearly demonstrated. Likewise, there is currently an unanswered question as to whether or not these LM deficits recover over time if the children are kept away from the noise source.

The relationship of noise and LM is currently a topic of considerable interest. In a recent study, Prior highlighted that there was a link between noise exposure and impairment of $\mathrm{LM}^{11}$. Some studies focused on this idea with different animal models, and they have documented the acute and chronic neurotoxic effects of noise stress. Furthermore, a study carried out on animals showed that chronic noise-induced oxidative stress may cause the 
impairment of memory, and reduce the dendritic count in the hippocampus ${ }^{12}$. In Kim's study, the influence of longterm noise on the memory and neurogenesis in the hippocampus of developing rats was investigated ${ }^{13)}$. In addition, a recent study reported the harmful effects of chronic exposure to noise on LM and also demonstrated that amino acid neurotransmitter levels and the expression of NMDA-R $\mathrm{B}$ were changed after noise exposure ${ }^{14)}$.

Although monoamine neurotransmitters play a key role in LM, little attention has been paid to their involvement in the deleterious effects of noise stress. LM is coordinated among different brain regions, especially the hippocampus and the medial prefrontal cortex ${ }^{12,15,16)}$. In Ravindran's study, rats were subjected to $100 \mathrm{~dB}$ broadband white noise for 15 days, which was found to alter the biogenic amine levels in discrete regions of the rat brain ${ }^{17)}$. However, to date, there is very little information about the noiseinduced changes of monoamine neurotransmitter levels in the hippocampus and there are no studies on long-term monoamine level alterations after noise exposure in the literature.

In some developing countries, especially in China, the apartments of workers are usually situated close to workplaces or city centers. Additionally, workers' children usually live together with their parents. Therefore, the noise exposure in the occupational environment is not only an issue for workers in industrial workplaces but also for children who live together with their parents in noisy occupational environments. The brain development of rats at 21 postnatal days is similar to that of a human toddler, while at 4-5 wk it is considered to be equivalent to that of an older, pre or peripubescent child. Taking account of this, we subjected 21-day-old rats to chronic noise exposure in the present study.

The aim of this study was to further examine whether the detrimental effects of noise stress on LM recover over time. In order to understand the potential mechanism of noise-induced LM deficits, we also studied the monoamine neurotransmitter levels in the hippocampus.

\section{Materials and Methods}

\section{Experimental animals}

Healthy male Sprague-Dawley rats $(\mathrm{N}=150$; weight $60-70 \mathrm{~g}$ ), at the age of 21 days old, obtained from Chongqing Medical University Lab Animal Center, were used in this study. The animals were maintained in a controlled environment with an air supply conditioned to a temperature of $25 \pm 1^{\circ} \mathrm{C}$ and relative humidity within 50 $\pm 10 \%$, and a $12 \mathrm{~h}$-light $/ 12 \mathrm{~h}$-dark cycle. The rats had free access to water and food in their home cages. Animals were given a one-week acclimation period to the laboratory condition. All procedures performed on the animals were approved by the ethics committee of Chongqing Medical University.

Before treatment, we used MWM to confirm that there was no significant difference in LM ability among the rats which were randomly chosen for the experiment. Animals were randomly assigned to three groups: (A) $80 \mathrm{~dB}$ noise exposure group, $\mathrm{n}=50$; (B) $100 \mathrm{~dB}$ noise exposure group, $\mathrm{n}=50$; (C) control group, $\mathrm{n}=50$. The 50 rats assigned to each group were further divided into 10 rats/group/time point: 1, 10, 20, 30, 40 days after discontinuation of noise exposure. Additionally, all efforts were made to minimize unwanted stress or discomfort to the animals during experimental procedures.

\section{Noise exposure}

In the present study, it was decided to expose rats to a representative noisy environment, the noise experienced on a Chinese aircraft carrier, similar to that recorded at a noisy airport environment. Therefore, the level of noise exposure in this study was set at $80 \mathrm{~dB}$. Likewise, the level of $100 \mathrm{~dB}$ was chosen as it is comparable with the noise levels detected in some industrial workplaces in China. The noise was designated environmental noise as it had a broad range of frequencies ranging from 250 to 2,000 Hz, similar to environmental noises. Broad-band white noise was produced by a noise generator (DF1681. Hangzhou Aihua instrument Co. China) and amplified by an amplifier, which was connected to a loudspeaker located $30 \mathrm{~cm}$ directly above the animal cage. In order to avoid circadian rhythm induced variation, treated animals were exposed to noise ( 80 or $100 \mathrm{~dB}, 4$ h per day for 30 days) at 09:00 to 13:00 daily and control animals were left undisturbed at the same time. Rats in the $80 \mathrm{~dB}, 100 \mathrm{~dB}$ and control group were separated in three experimental rooms (size: $6 \mathrm{~m} \times 5 \mathrm{~m} \times 3 \mathrm{~m}$ ). Animals were kept in the wire mesh cages, with one animal per cage (size: $46 \mathrm{~cm}$ $\times 34 \mathrm{~cm} \times 20 \mathrm{~cm}$ ), placed in the center of the sound field. The intensity of the sound, $80 \mathrm{~dB}$ or $100 \mathrm{~dB}$, distribution in the cage was measured by a precision sound level meter (AWA6218C. Hangzhou Aihua instrument Co. China). The noise level variation was approximately $2 \mathrm{~dB}$ and the background noise level in the chamber was below $30 \mathrm{~dB}$. Further, multiple distant cues around the room were kept in the same places.

\section{Behavioral testing}

LM was tested using MWM. The water maze consisted of a pool with a diameter of $130 \mathrm{~cm}, 50 \mathrm{~cm}$ high, filled with tap water to a depth of $30 \mathrm{~cm}$, adjusted to $22 \pm 1^{\circ} \mathrm{C}$ and made opaque by the addition of powdered white nontoxic tempera paint. The pool was placed in a $2.5 \times 3 \times$ 2.5 rectangular room containing invariant spatial stimuli. A circular escape platform $9 \mathrm{~cm}$ in diameter was placed 1 $\mathrm{cm}$ below the surface of the water in the middle of one of the four quadrants of the pool, and this position remained constant throughout testing. A video camera suspended from the ceiling above the center of the pool was used to monitor the animals' behavior. All animals were allowed 
to circumnavigate the pool in search of the escape platform for a maximum of $60 \mathrm{~s}$. Place training consisted of four trials each day for 4 consecutive days. In each trial, a rat was lowered by hand into the pool and placed in the water facing the inside wall of the pool at one of four start positions that were spaced equally around the rim of the pool. The start points used for each trial varied in a pseudo-random sequence. Latency of reaching the escape platform was recorded. If a rat did not reach the platform within $60 \mathrm{~s}$, it was guided to the platform by the experimenter, left on the platform for $10 \mathrm{~s}$, and assigned a latency score of $60 \mathrm{~s}$ for that trial. Animals were returned to a holding cage for $60 \mathrm{~s}$ between trials. On the final trial of day 5 the rats were given a 60 -second retention test of the location with the platform absent. The numbers of crossing of the exact location of the platform and the time spent in the target quadrant were recorded.

\section{Monoamine neurotransmitter assays}

At the end of each behavioral test, the animals were sacrificed by cervical dislocation under carbon dioxide anesthesia. The hippocampus was collected, and the concentration of monoamine neurotransmitters, including DA, NE, and 5-HT were measured using a HPLC-EC (high pressure liquid chromatography with electrochemical detection). The specimens were homogenized in $0.1 \mathrm{M}$ $\mathrm{HClO}_{4}$. Then, homogenates were centrifuged for $15 \mathrm{~min}$ at $15,000 \mathrm{r} / \mathrm{min}$, supernatants were filtered through $20 \mu \mathrm{l}$ filters and analyzed by HPLC-EC. The chromatograph used was an ESA CoulArray 5600A with a MD-150 column and a mobile phase of $60 \mathrm{mM} \mathrm{NaH} \mathrm{PO}_{2}, 1.5 \mathrm{mM}$ octyl sulfonate, $50 \mu \mathrm{M}$ EDTA and $9 \%$ acetonitrile, at a $\mathrm{PH}$ of 3.5 and a flow rate of $0.6 \mathrm{ml} / \mathrm{min}$ with voltages of $\mathrm{CHI}:-100 \mathrm{mV}, \mathrm{CH} 2$ : +100 mV, CH3: +300 mV, CH4: +500 mV.

\section{Statistics analysis}

Quantitative data are expressed as mean \pm S.D. unless otherwise indicated. Data were analyzed using SPSS 11.0. Statistical significance was assessed with repeated measures ANOVA and one-way ANOVA, and values of $p<0.05$ were considered significant. The StudentNewman-Keuls multiple comparison test was used to compare pairs of means; $p<0.05$ was considered to indicate statistical significance.

\section{Results}

\section{The effect of noise exposure on LM}

In the MWM test, compared to the controls, the noisestress exposed animals showed significant increases in escape latency (Fig. $1 ; p<0.05$ ) and their the frequency of crossing the platform and time spent in the target quadrant were significantly decreased (Fig. 2 and Fig. 3 ; $p<0.05$ ). We also found the performance of the young male rats in the $80 \mathrm{~dB}$ exposure group was significantly better than that of $100 \mathrm{~dB}$ group $(p<0.05)$. However, we found that

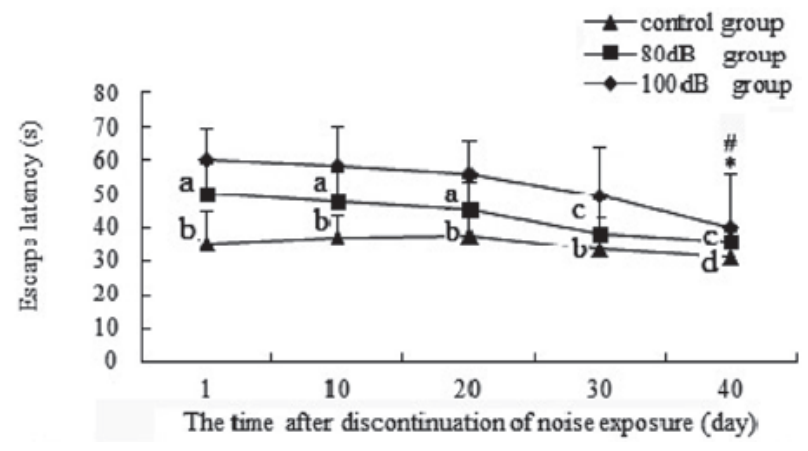

Fig. 1. The effect of noise exposure on escape latency in MWM. Escape latency, in seconds, to reach the platform during each trial. Values were mean \pm S.D. of escape latency made on different days. A repeated measures ANOVA found that there was a significant difference in the escape latencies of different noise level $(\mathrm{F}=133.07$, $p=0.000$ ). After discontinuation of noise exposure, there were significant differences in the escape latencies at different time periods $(\mathrm{F}=29.07, p=0.000)$. There was a significant interaction between the level of noise and time point $(\mathrm{F}=4.90, p=0.000)$. *: There was no significant difference in the escape latencies of the control group with time $(\mathrm{F}=0.628, p=0.645>0.05)$. \#: After discontinuation of noise exposure, the escape latency decreased significantly in the $80 \mathrm{~dB}$ and $100 \mathrm{~dB}$ groups with time $(\mathrm{F}=3.26, p=0.020<0.05 ; \mathrm{F}=3.95$, $p=0.008<0.05$ ). a: $80 \mathrm{~dB}$-exposed animals showed a significant increase compared to the control at the indicated times, $p<0.05$; b: $100 \mathrm{~dB}$-exposed animals showed a significant increase compared to the control and $80 \mathrm{~dB}$ group at the indicated times, $p<0.05$; c: 80 $\mathrm{dB}$-exposed animals showed no significant difference from the control group at the indicated times, $p>0.05$; : $100 \mathrm{~dB}$-exposed animals showed no significant difference from the control and $80 \mathrm{~dB}$ group at the indicated times, $p>0.05$.

the escape latency of the noise-exposed rats was decreased significantly with time from cessation of noise exposure (Fig. $1 ; p<0.05$ ), and their frequency of crossings and time spent in the target quadrant increased with the passage of time from cessation of noise exposure (Fig. 2 and Fig. 3; $p<0.05)$. The performance of the $80 \mathrm{~dB}$ group had improved to that of control by day $30(p>0.05)$, and the performance of the $100 \mathrm{~dB}$ group was not different from those of the $80 \mathrm{~dB}$ group or control by day 40 after cessation of noise exposure $(p>0.05)$.

\section{Changes of monoamine neurotransmitters}

As shown in Fig. 4-6, our results demonstrate that the levels of DA, NE and 5-HT were significantly decreased in the hippocampus of young male rats after noise exposure compared to the controls (Fig. 4, Fig. 5 and Fig. 6; $p<0.05$ ). We also found the levels of DA, NE and 5-HT in the 80 


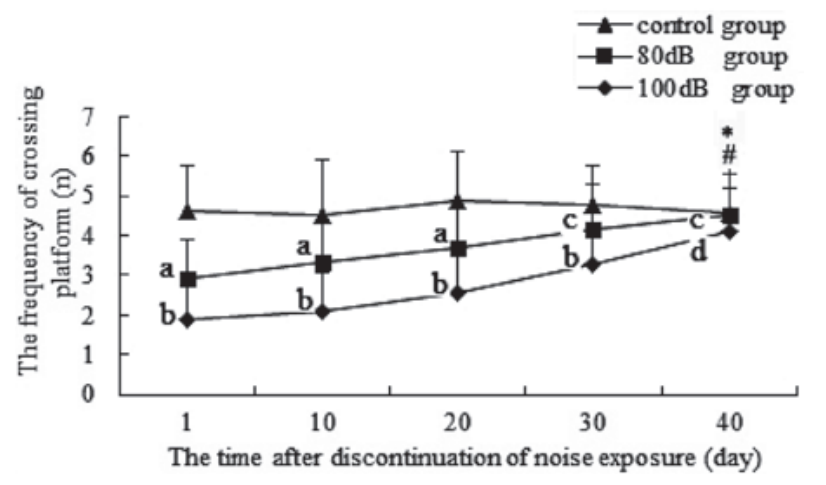

Fig. 2. The effect of noise exposure on the frequency of crossing the platform in MWM. Values were mean \pm S.D. of the frequency of crossing the platform on different days. A repeated measures ANOVA found that there was a significant difference in the frequencies of crossing the platform at different noise exposures $(\mathrm{F}=51.84, p=0.000)$. After discontinuation of noise exposure, there were significant differences on the frequencies of crossing the platform at different times $(\mathrm{F}=9.33, p=0.000)$. There was a significant interaction between the level of noise and time $(\mathrm{F}=2.53, p=0.021)$. $*$ : There was no significant difference in the frequency of the crossing platform in the control group with time $(\mathrm{F}=0.175, p=0.950>0.05)$. \#: After discontinuation of noise exposure, the frequency of the crossing platform increased significantly in the $80 \mathrm{~dB}$ and $100 \mathrm{~dB}$ groups with time $(\mathrm{F}=3.17, \quad p=0.022<0.05 ; \mathrm{F}=8.04$, $p=0.000<0.05$ ). a: $80 \mathrm{~dB}$-exposed animals showed a significant decrease compared to the control at the indicated times, $p<0.05$; b: $100 \mathrm{~dB}$-exposed animals showed a significant decrease compared to the control and $80 \mathrm{~dB}$ group at the indicated times, $p<0.05$; $\mathrm{c}: 80$ $\mathrm{dB}$-exposed animals showed no significant difference from the control group at the indicated times, $p>0.05$; $\mathrm{d}$ : $100 \mathrm{~dB}$-exposed animals showed no significant difference from to the control and $80 \mathrm{~dB}$ group at the indicated times, $p>0.05$.

$\mathrm{dB}$ exposure group were significantly higher than those in the $100 \mathrm{~dB}$ group $(p<0.05)$. However, we found that after discontinuation of noise exposure, the levels of DA, NE and 5-HT increased in the two exposed groups with the passage of time from cessation of noise exposure, and those of the $80 \mathrm{~dB}$ group had improved to control levels by day $30(p>0.05)$, and those of the $100 \mathrm{~dB}$ group did not differ from those of the $80 \mathrm{~dB}$ group or control by day 40 after cessation of noise exposure $(p>0.05)$.

\section{Discussion}

Noise is a ubiquitous environmental stress. Chronic noise stress was found to damage cognition in a number of ways, such as acquisition of memory, attention, and reading comprehension and recall ${ }^{2,18-21)}$. Noise intensity used in the study of effects of noise on functions of the

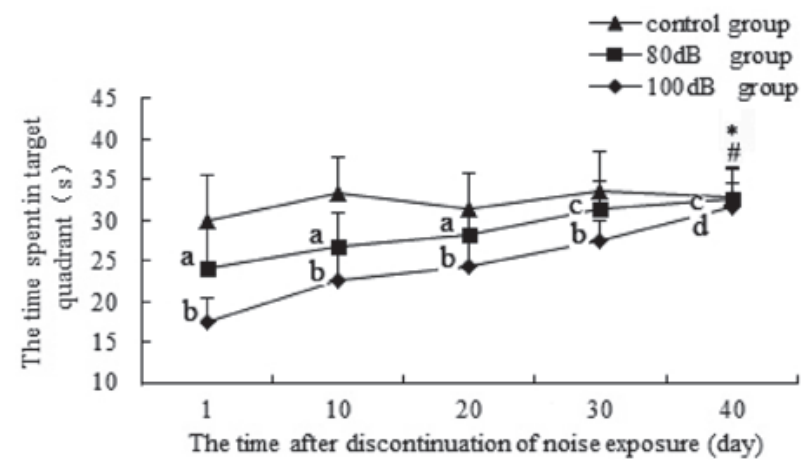

Fig. 3. The effect of noise exposure on the time spent in the target quadrant in MWM. Values were mean \pm S.D. of the time spent in the target quadrant on different days. A repeated measures ANOVA found that there was a significant difference in the times spent in the target quadrant after the different noise exposures $(\mathrm{F}=21.58$, $p=0.000$ ). After discontinuation of noise exposure, there were significant differences in the times spent in the target quadrant at different times $(\mathrm{F}=15.52, p=0.000)$. There was a significant interaction between the level of noise and time $(\mathrm{F}=3.31, p=0.004)$. *: There was no significant difference in the time spent in the target quadrant in the control group with time $(\mathrm{F}=1.29$, $p=0.284>0.05$ ). \#: After discontinuation of noise exposure, the time spent in the target quadrant increased significantly in the $80 \mathrm{~dB}$ and $100 \mathrm{~dB}$ groups with time $(\mathrm{F}=8.77, p=0.000<0.05 ; \mathrm{F}=41.77, p=0.000<0.05)$. a: 80 $\mathrm{dB}$-exposed animals showed a significant decrease compared to the control at the indicated times, $p<0.05$; b: $100 \mathrm{~dB}$-exposed animals showed a significant decrease compared to the control and $80 \mathrm{~dB}$ group at the indicated times, $p<0.05$; $\mathrm{c}: 80 \mathrm{~dB}$-exposed animals showed no significant difference from the control group at the indicated times, $p>0.05$; $\mathrm{d}: 100 \mathrm{~dB}$-exposed animals showed no significant difference from the control and $80 \mathrm{~dB}$ group at the indicated times, $p>0.05$.

central nervous system has typically ranged from $80-120$ $\mathrm{dB}^{14,22-24)}$. In this study, we chose $80 \mathrm{~dB}$ and $100 \mathrm{~dB}, 4 \mathrm{~h}$ per day for 30 days as chronic noise, with reference to previous studies ${ }^{12,14)}$ and our experimental experience. Noise pollution is a widespread and growing problem in China and the levels used in our study were near to those commonly experienced in China.

The Morris water maze was designed by the English psychologist Morris in the 1980s. The place navigation and probe test are considered to be the most specific tests for spatial LM. From the results of the MWM tests presented in this study, we conclude that chronic noise exposure induces adverse effects on LM. Our results are in agreement with those of Manikandan and Cui ${ }^{12,14)}$. We also found that the performances of young male rats in the $80 \mathrm{~dB}$ exposure group were significantly better than those 


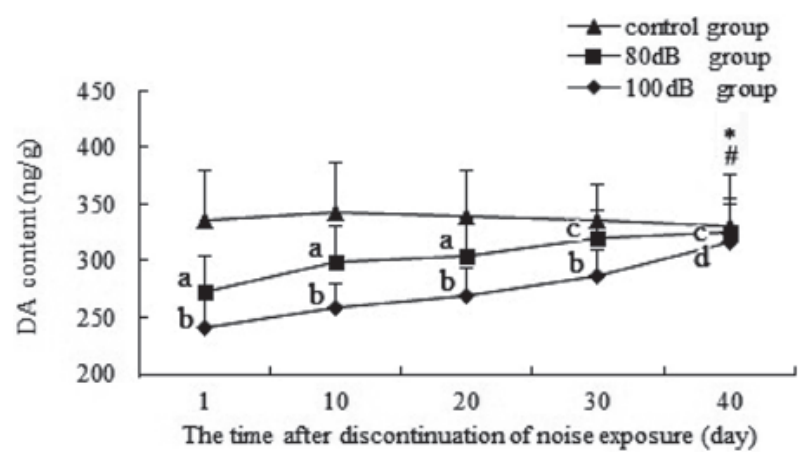

Fig. 4. The effect of noise exposure on the level of DA. Values were mean \pm S.D. of DA on different days. A repeated measures ANOVA found that there was a significant difference in DA after different noise exposures $(\mathrm{F}=43.89, p=0.000)$. After discontinuation of noise exposure, there were significant differences in DA at different times $(\mathrm{F}=7.73, p=0.000)$. There was a significant interaction between the level of noise and time $(\mathrm{F}=2.80, p=0.010)$. *: There was no significant difference in the level of DA in the control group with time $(\mathrm{F}=0.128, p=0.972>0.05)$. \#: After discontinuation of noise exposure, the level of DA increased significantly in the $80 \mathrm{~dB}$ and $100 \mathrm{~dB}$ groups with time $(\mathrm{F}=6.78$, $p=0.000<0.05 ; \mathrm{F}=11.94, p=0.000<0.05)$. a: $80 \mathrm{~dB}$ exposed animals showed a significant decrease compared to the control at the indicated times, $p<0.05$; b: $100 \mathrm{~dB}$-exposed animals showed a significant decrease compared to the control and $80 \mathrm{~dB}$ group at the indicated times, $p<0.05$; $c$ : $80 \mathrm{~dB}$-exposed animals showed no significant difference from the control group at the indicated times, $p>0.05$; $: 100 \mathrm{~dB}$-exposed animals showed no significant difference from to the control and $80 \mathrm{~dB}$ group at the indicated times, $p>0.05$.

of the $100 \mathrm{~dB}$ group, suggesting that noise intensity may be related to the level of impairment ${ }^{25}$. However, in the MWM, after the discontinuation of noise exposure, both the $80 \mathrm{~dB}$ and $100 \mathrm{~dB}$ groups showed no significant differences from the control group 30 and 40 days after cessation of noise exposure, respectively. This recovery suggests that the noise-induced impairment was only transient and that the $80 \mathrm{~dB}$ and $100 \mathrm{~dB}$ noise exposures of 30 days did not cause long-term damage to LM. However, to date, little is known about this recovery, and further investigation is warranted.

Monoamine neurotransmitters are very important for cognitive function. Increases in the expression of monoamine neurotransmitters is hypothesized to improve cognitive impairment. Furthermore, both NE and 5-HT promote specific biochemical and electrophysiological changes that may critically underlie LM. Likewise, DA disorders in the hippocampus can cause a decline in

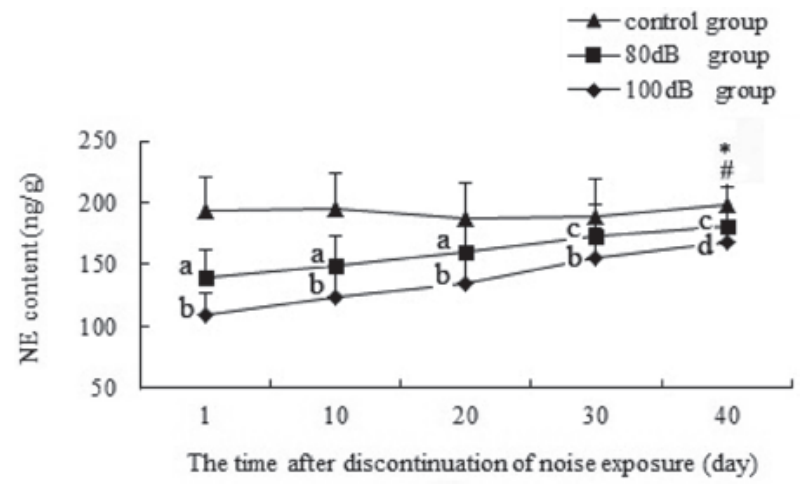

Fig. 5. The effect of noise exposure on the level of NE. Values were mean \pm S.D. of NE on different days. A repeated measures ANOVA found that there was a significant difference in NE after the different noise exposures $(\mathrm{F}=65.17, p=0.000)$. After discontinuation of noise exposure, there was a significant difference in NE at different times $(\mathrm{F}=11.30, p=0.000)$. There was a significant interaction between the level of noise and time $(\mathrm{F}=3.07, p=0.005)$. *: There was no significant difference in the level of NE in the control group with time $(\mathrm{F}=0.278, p=0.891>0.05)$. \#: After discontinuation of noise exposure, the level of NE increased significantly in the $80 \mathrm{~dB}$ and $100 \mathrm{~dB}$ groups with time $(\mathrm{F}=5.34$, $p=0.001<0.05 ; \mathrm{F}=17.66, p=0.000<0.05)$. a: $80 \mathrm{~dB}-$ exposed animals showed a significant decrease compared to the control at the indicated times, $p<0.05$; b: $100 \mathrm{~dB}$-exposed animals showed a significant decrease compared to the control and $80 \mathrm{~dB}$ group at the indicated times, $p<0.05$; $c: 80 \mathrm{~dB}$-exposed animals showed no significant difference from the control group at the indicated times, $p>0.05$; d: $100 \mathrm{~dB}$-exposed animals showed no significant difference from the control and $80 \mathrm{~dB}$ group at the indicated times, $p>0.05$.

neurocognitive functions, especially those related to memory, attention, and problem-solving. Czyrak's study confirmed that monoamine neurotransmitters play an essential role in LM and are also involved in memory retrieval $^{26)}$. Moreover, alterations in brain monoamine neurotransmitter release are associated with brain injury and cognitive impairment ${ }^{27}$. Acute or sub-chronic noise exposures were both found to change the level of biogenic amines in discrete regions of the rat brain ${ }^{17,26)}$. Our results demonstrat that the levels of DA, NE and 5-HT were significantly decreased in the hippocampi of young male rats after noise exposure (Fig. 4-6), and are in agreement with the results of previous studies. We also found that the levels of DA, NE, 5-HT in the $80 \mathrm{~dB}$ group were significantly higher than those in the $100 \mathrm{~dB}$ group. These results suggest that the decreased concentrations of DA, $\mathrm{NE}$ and 5-HT in the hippocampi of the young rats exposed to noise may have influenced the plasticity of the synapses 


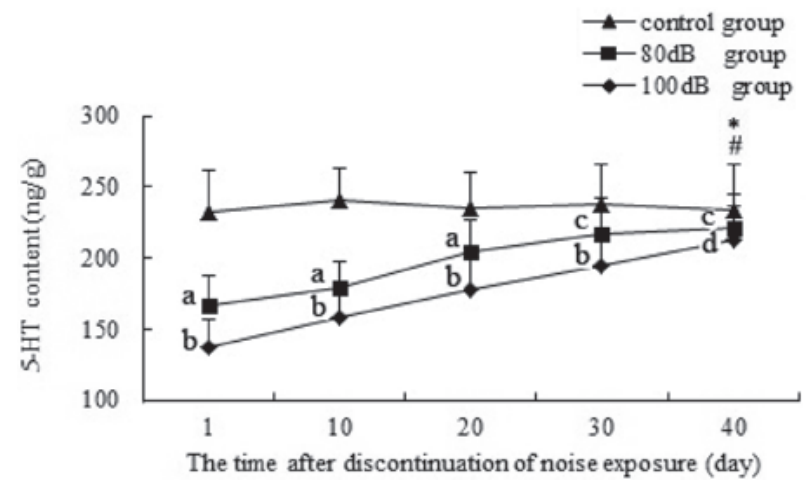

Fig. 6. The effect of noise exposure on the level of 5-HT. Values were mean \pm S.D. of 5-HT on different days. A repeated measures ANOVA found that there was a significant difference on 5-HT after different noise exposures $(\mathrm{F}=112.93, p=0.000)$. After discontinuation of noise exposure, there were significant differences in 5-HT at different times $(\mathrm{F}=15.48, p=0.000)$. There was a significant interaction between the level of noise and time $(\mathrm{F}=4.30, p=0.000)$. *: There was no significant difference in the level of 5-HT in the control group with time $(\mathrm{F}=0.128, p=0.972>0.05)$. \#: After discontinuation of noise exposure, the level of 5-HT increased significantly in the $80 \mathrm{~dB}$ and $100 \mathrm{~dB}$ groups with time $(\mathrm{F}=11.41, p=0.000<0.05 ; \mathrm{F}=19.76, p=0.000<0.05)$. a: $80 \mathrm{~dB}$-exposed animals showed a significant decrease compared to the control at the indicated times, $p<0.05$; b: $100 \mathrm{~dB}$-exposed animals showed a significant decrease compared to the control and $80 \mathrm{~dB}$ group at the indicated times, $p<0.05$; c: $80 \mathrm{~dB}$-exposed animals showed no significant difference from the control group at the indicated times, $p>0.05$; $: 100 \mathrm{~dB}$-exposed animals showed no significant difference from the control and $80 \mathrm{~dB}$ group at the indicated times, $p>0.05$.

resulting in impairment of LM. This interpretation is consistent with results showing that stress impaired working memory performance in rats and monkeys ${ }^{28,29)}$. Therefore, it is logical to postulate that changes in the hippocampus, especially the levels of monoamine neurotransmitters and their receptors, are important mechanisms underlying the chronic noise-induced LM deficits. This has not received much attention in the literature. Only Arnsten and McDonald have investigated DA and 5- $\mathrm{HT}_{2 \mathrm{~A}}$ receptors in the medial prefrontal cortex and their roles in mediating the effects of chronic stress ${ }^{18,30)}$. In the present study, the levels of DA, NE and 5-HT increased from the 1 to the 40 days after cessation of noise exposure in both the noise-exposed groups, and the performance of the $80 \mathrm{~dB}$ group in the MWM reached that of the control by day 30 whilst that of the $100 \mathrm{~dB}$ group was no different from those of the 80 $\mathrm{dB}$ and control groups by day 40 , suggesting the impairment of LM recovers over time. These results also show that the effect of chronic noise stress on LM deficits of young male rats may be related to the decrease of monoamine neurotransmitters in the hippocampus ${ }^{31}$.

\section{Conclusion}

The results of this work indicate that LM deficits following chronic exposure to noise are related to monoamine neurotransmitter level alterations in young male rats. Moreover, our findings show that noise-induced LM deficits in young male rats and reduced monoamine neurotransmitters in the hippocampus recover over time. Furthermore, these outcomes suggest that chronic exposure to noise would have similar effects on children's physiology and behavior.

Acknowledgment: This work was supported by the Foundation of Health Department of Sichuan Province. NO: 070228.

\section{References}

1) Babisch W. Noise/stress concept, risk assessment and research needs. Noise Health 2002; 4: 1-11.

2) Birgitta B, Thomas L, Dietrich HS. Guidelines for community noise. Singapore: Institute of Environmental Epidemiology, Ministry of Environment; 2000. p.2536.

3) Ising H, Kruppa B. Health effects caused by noise: evidence in them literature from the past 25 years. Noise Health 2004; 6: 5-13.

4) Smith A. A review of the effects of noise on human performance. Scand J Psychol 1989; 30: 185-206.

5) Haines MM, Stansfeld SA, Job RF, Berglund B, Head J. Chronic aircraft noise exposure, stress responses, mental health and cognitive performance in school children. Psychol Med 2001; 31: 265-77.

6) Matsui T, Stansfeld S, Haines M, Head J. Children's cognition and aircraft noise exposure at home-the West London Schools. Noise Health 2004; 7: 49-57.

7) Stansfeld S, Berglund B, Clark C, et al. Aircraft and road traffic noise and children's cognition and health: a cross-national study, Lancet 2005; 365: 1942-9.

8) Clark C, Martin R, Kempen E, et al. Exposure-effect relations between aircraft and road traffic noise exposure at school and reading comprehension: the RANCH project. Am J Epidemiol 2006; 163: 27-37.

9) Haines MM, Stansfeld SA, Job RF, Berglund B, Head J. A follow-up study of effects of chronic aircraft noise exposure on child stress responses and cognition. Int J Epidemiol 2001; 30: 839-45.

10) Stansfeld S, Haines M, Brown B. Noise and health in the urban environment. Rev Environ Health 2000; 15: 43-82.

11) Prior H. Effects of predictable and unpredictable intermittent noise on learning in rats. Behav Brain Res 2002; 133: 117-24.

12) Manikandan S, Padmab MK, Srikumar R, Parthasarathy NJ, Muthuvel A, Devi RS. Effects of chronic noise stress on memory of rats in relation to neuronal dendritic 
alteration and free radical-imbalance in hippocampus and medial prefrontal cortex. Neurosci Let 2006; 399: $17-22$.

13) Kim $\mathrm{H}$, Lee MH, Chang HK, et al. Influence of prenatal noise and music on the memory and neurogenesis in the hippocampus of developing rats. Brain Dev 2006; 28 : 109-14.

14) Cui B, Wu MG, She XJ. Effects of chronic noise exposure on spatial learning and memory of rats in relation to neurotransmitters and NMDAR2B alteration in the hippocampus. J Occup Health 2009; 51: 152-8.

15) Diorio V, Viau MJ, Meaney. The role of the medial prefrontal cortex (cingulate gyrus) in the regulation of hypothalamic-pituitary-adrenal responses to stress. J Neurosci 1993; 13: 3839-47.

16) Lupien SJ, Lepage M. Stress, memory, and the hippocampus: can't live with it, can't live without it. Behav Brain Res. 2001; 127: 137-58.

17) Ravindran R, Devi RS, Samson J, Senthilvelan M. Noise-stress-induced brain neurotranamitter changes and the effect of Ocimum sanctum (Linn) treatment in albino rats. J Pharmacol Sci 2005; 98: 354-60.

18) Arnsten AF, Goldman RP. Noise stress impairs prefrontal cortical cognitive function in monkeys: evidence for a hyperdopaminergic mechanism. Arch Gen Psychiatry 1998; 55: 362-8.

19) Hygge S, Boman E, Enmarker I. The effects of road traffic noise and meaningful irrelevant speech on different memory systems. Scand J Psychol 2003; 44: $13-21$.

20) Hygge S, Evans GW, Bullinger M. A prospective study of some effects of aircraft noise on cognitive performance in school children. Psychol Sci 2002; 13: 469-74.

21) Pavlides C, Nivon LG, McEwen BS. Effects of chronic stresson hippocampal long-term potentiation. Hippocampus 2002; 12: 245-57.

22) Griefahn B. Sleep disturbances related to environmental noise. Noise Health 2002; 4: 57-60.

23) Lemaire V, Koehl M, Le Moal M, Abrous DN. Prenatal stress produces learning deficits associated with an inhibition of neurogenesis in the hippocampus. Proc Natl Acad Sci USA 2000; 97: 11032-7.

24) Muzet A. The need for a specific noise measurement for population exposed to aircraft noise during night-time. Noise Health 2002; 4: 61-4.

25) Job RF. Noise sensitivity as a factor influencing human reaction to noise. Noise Health 1999; 1: 57-68.

26) Czyrak A, Mackowiak M, Chocyk A, Fijal K, Wedzony $\mathrm{K}$. Role of glucocorticoids in the regulation of dopaminergic neurotransmission. Pol J Pharmacol 2003; 55: 667-74.

27) Manikandan S, Srikumar R, Parthasarathy NJ, Devi RS. Protective effect of Acorus calamus LINN on free radical scavengers and lipid peroxidation in discrete regions of brain against noise stress exposed rat. Biol Pharm Bull 2005; 28: 2327-30.

28) Murphy BL, Arnsten AF, Jentsch JD, Roth RH. Dopamine and working memory in rats and monkeys: pharmacological reversal of stress-induced impairment. J Neurosci 1996; 16: 7768-75.

29) Murphy BL, Arnsten AF, Goldman RP, Roth RH. Increased dopamine turnover in the prefrontal cortex impairs working memory performance in rats and monkeys. Proc Natl Acad Sci USA 1996; 93: 1325-9.

30) McDonald LM, Moran PM, Vythelingum GN, Joseph $\mathrm{MH}$, Stephenson JD, Gray JA. Latent inhibition is attenuated by noise and partiallyrestored by a 5-HT2A receptor antagonist. Behav Pharmacol 2002; 13: 6637.

31) Tsai HY, Lu YH, Wu CR, Chen YF. Effects of noise on monoamine levels in the rat brain using in vivo microdialysis. Pflugers Arch Eur J Phy 2005; 450: 83-7. 